\title{
PAGET'S DISEASE OF THE EYELID ASSOCIATED WITH CARCINOMA
}

\author{
BY \\ DR. A. HAGEDOORN \\ (UNIVERSITY EYE hOSPITAL, AMSTERDAM)
}

IN September, 1929, B. P., a woman aged 56 years was sent to the clinic by her ophthalmologist on account of severe pain in the left eye. She had been under treatment for trachoma since childhood. The left tarsus was extirpated in 1921 and the right lacrimal sac in 1925. She suffered from recurrent attacks of iritis in the left eye. The right eye showed cicatricial trachoma, pannus, and no sign of irritation or inflammation. There was myopia of 1 dioptre, vision after correction being $1 / 6$.

The left eye had blepharitis, dacryocystitis and there was a thick viscous secretion. The conjunctiva was thick and reddened, the surface appearing a little " greasy," the conjunctival sac was considerably narrowed by cicatrices. The surface of the cornea was irregular and there was a dense old pannus. As there was light perception and normal tension the sac was extirpated, further treatment being conservative. After two years, however, it proved necessary to excise the eye together with the posterior part of the lids and the conjunctival sac as the socket would have been too small for the reception of an artificial eye.

In examining the sections of this case the following changes were found:-

(a) Changes due to chronic trachoma:-Xerosis of the conjunctiva, with production of keratohyaline and horn in the superficial layers of the epithelium. It is impossible to trace the place where surface epithelium and conjunctival epithelium join. Changes in the normal anatomy. In some sections a fairly normal tarsus with normal Meibomian glands is present, so that the tarsus must have been removed only partially. In most sections, however, smaller and larger vessels have grown into the tarsal tissue so that the margin of the eyelid cannot be recognised. The ducts of the glands open on the conjunctival side of the eyelid (entropion). Obliteration of glands and occasionally a cyst were seen. Inflammatory changes. Infiltration with lymphocytes, plasma cells, rarely in follicles or perivascular, but especially under the xerotic epithelium.

(b) Besides the above there were some interesting anomalies of a blastomatous nature. As stated above, instead of the normal conjunctival epithelium, a stratified epithelium covers the posterior surface of the eyelid (xerosis). It is clearly marked off 
against the underlying tissue by a basal membrane. On top of the regular basal cells there are a varying number of layers, constituting the stratum spinosum, the spinae of which are difficult to see in the haematoxylin-eosin and v. Gieson stain. The superficial layers may contain keratohyaline. Generally a thin stratum corneum is present, sometimes even without an underlying layer of keratohyaline containing cells. In studying this epithelium from

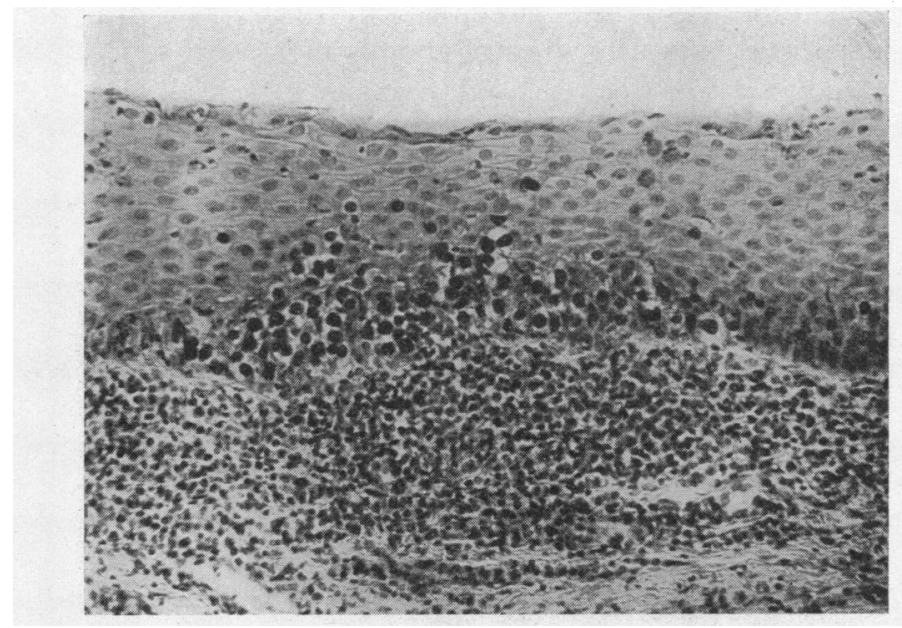

FIG. 1.

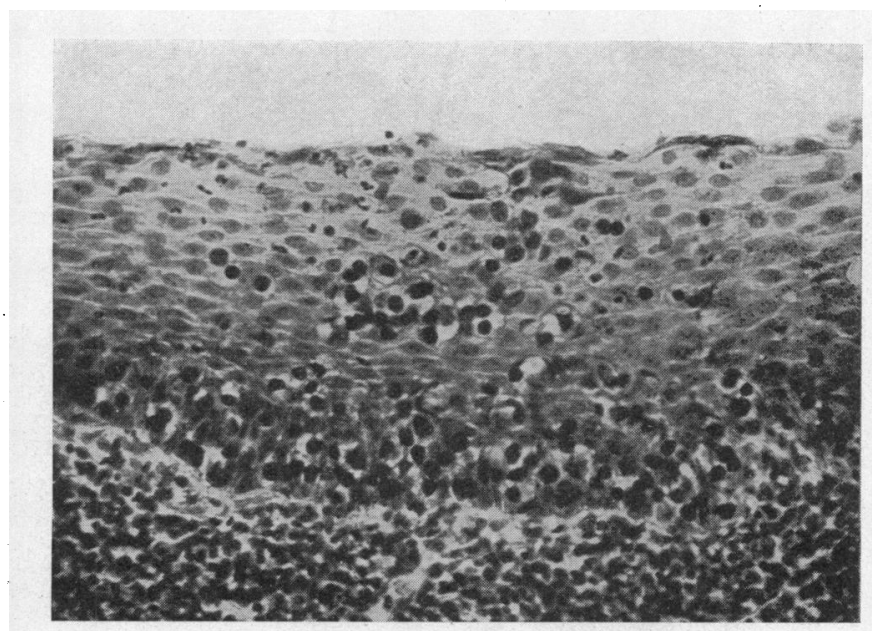

FIG. 2. 
the cornea towards the margin of the lid, though varying in thickness, it shows a regular structure. Then, at the posterior surface of the tarsus swollen hydropic cells appear. These cells of abnormal shape constitute a layer of many cells' thickness chiefly in the basal cell region (Fig. 1), but they may be present in the higher layers as well (Fig. 2). In some sections even, areas of epithelium may be found which are entirely altered in their whole thickness, the normal cells having disappeared on account of the hydropic cells. The nucleus of these cells is nearly round, the chromatin network and the nucleoli have been transformed into a dense darkly staining homogeneous mass (Figs. 1, 2). Here

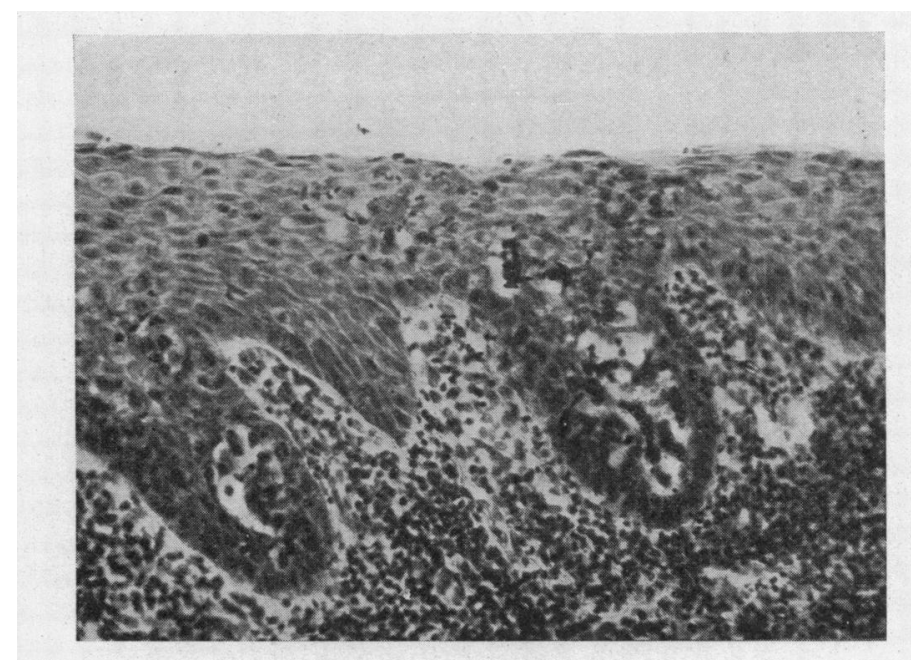

FIG. 3.

and there are seen nuclei, staining less intensively with a trace of intra-nuclear structure, irregular nuclei, nuclei resembling those of the basal cells and prickle cells surrounded by a hydropic, vacuolated protoplasm, which often seemed to be entirely absent, the nucleus lying in an empty space marked off often by a distinct cellular wall, showing no trace of prickles. Though in manipulating with the microscrew of the microscope pathological nuclei rarely seem to be flat, and crescent shaped nuclei are rare. Occasionally pyknotic, darkly staining nuclei clump together. Besides the vacuoles in the cells themselves there are empty spaces between them simulating an intercellular oedema, while in other areas these spaces seem to have originated by confluence of hydropic cells (Fig. 3). It is extremely rare to meet a cell near the superficial layers which shows keratinisation. 
Typical areas of xerotic epithelium alternate with diseased areas probably on account of a net-like distribution of the affection under the epithelium. It was also found in the epithelium of the inferior eyelid and continuous with the diseased areas of the superior eyelid at the canthus. It proved impossible to make a reliable reconstruction as more or less abnormal cells were found in apparently healthy epithelium. Either these hydropic cells must have a peculiar faculty of creeping under the deeper layers of the epithelium and in the epithelium over a large distance, without passing through the basal membrane, or else they must have developed from multiple centres.

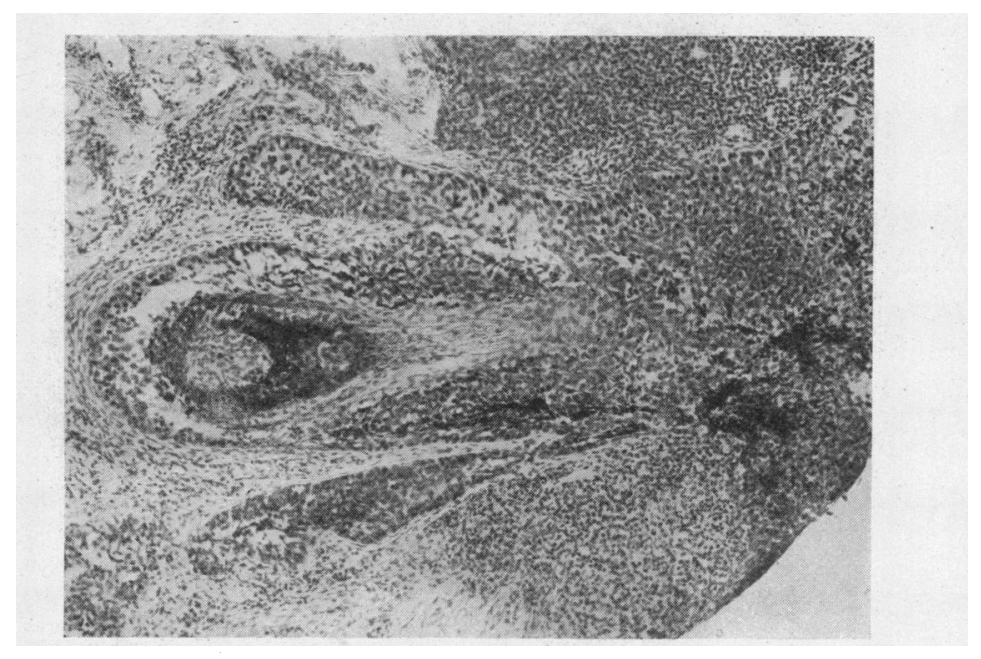

FIG. 4.

Towards the margin of the lid the epithelium is thicker with a well developed stratum granulosum and stratum corneum. The degeneration of the epithelium almost exclusively concerns the deeper layers, but here also affects the glands opening out on the surface. The cells surrounding the hairs and of the associated glands show the same vacuolated hydropic aspect as the pathological cells described under the xerotic epithelium. However, the nuclei are generally not so deeply stained and irregular, the empty inter-cellular spaces are often considerable. It seemed very doubtful whether this had to be considered a degeneration caused by a prolonged chronic inflammation or an affection of a blastomatous nature (Fig. 4). However, the cells completely filling up a Meibomian gland lie close together, the protoplasm stains well, the shape of the darkly stained nuclei 
varies considerably from round to elongated and thin, strongly resembling certain varieties of basal cell carcinoma. Still it seemed as if these cells did not grow infiltratively (carcinoma in situ?) (Fig. 5). On carefully inspecting the sections, however, a region could be found with infiltrative growth of similar cells (Fig. 6). Carcinoma seemed probable.

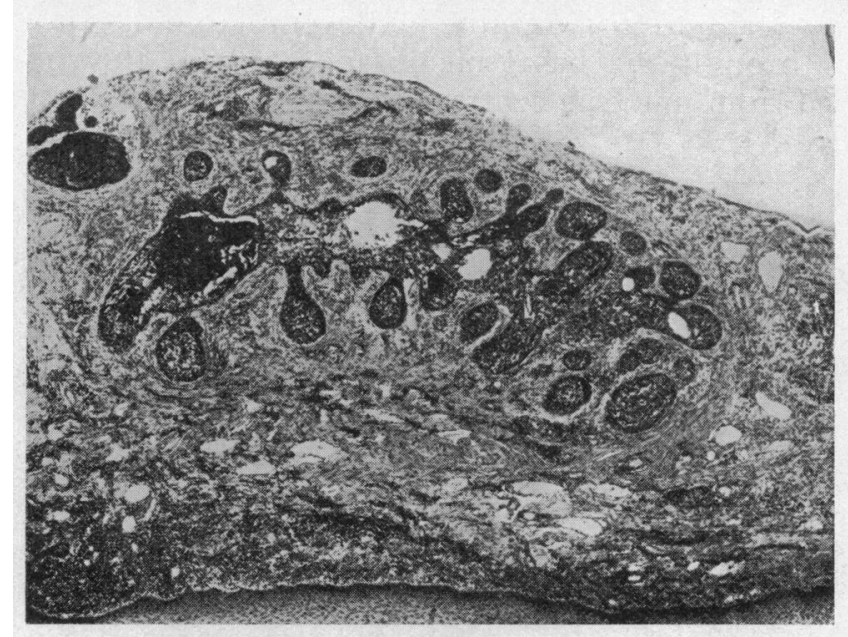

FIG. 5.

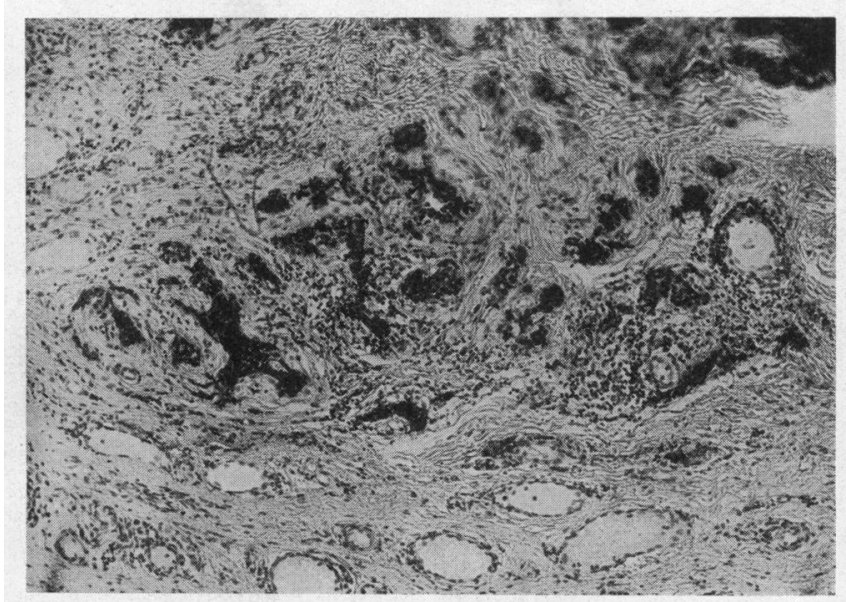

FIG. 6. 
One year later the patient re-entered the clinic, as she had observed a small tumour under the upper eyelid. As this recurrence confirmed the diagnosis of carcinoma I performed exenteration of the orbit. At the present date she has had no recurrence.

On general examination no other primary malignant tumour or metastasis could be detected. She had been under treatment since the age of 25 years. In 1903 she was three months in the University Hospital for Nervous Diseases; in 1904 palsy of both legs and the left arm; in 1925 palsy of the right arm; and in 1924

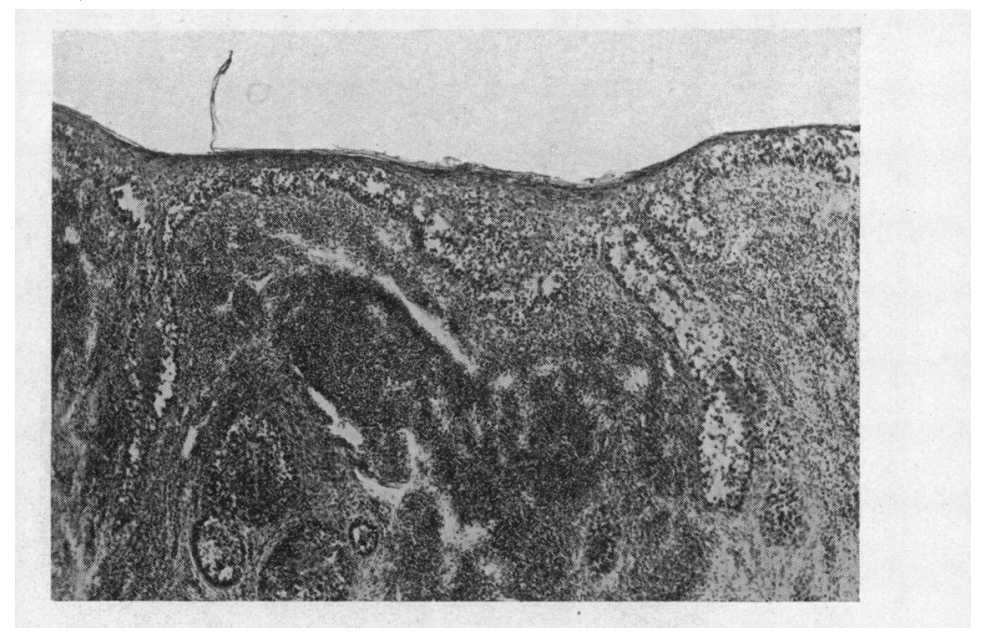

FIG. 7.

an operation was performed on the stomach. A complicated case history, which does not make a primary tumour elsewhere probable. She was affected with hysteria.

Material of the exenteration showed a similar affection of the skin, which was quite normal in the non-affected regions. Here again the anomaly is chiefly present in the deeper layers of the epithelium, though here and there it may be affected throughout its whole thickness (Fig. 7). Darkly staining nuclei are rather rare, whereas the vacuolisation of the protoplasm of the cells is very considerable.

The nuclei are of a varying shape and irregularly arranged even in the higher layers. Large cells with a crescent-shaped nucleus were more frequent here than in the first described affection of the conjunctiva. Isolated pathological cells are found in the normal epithelium at a great distance from the diseased areas. 


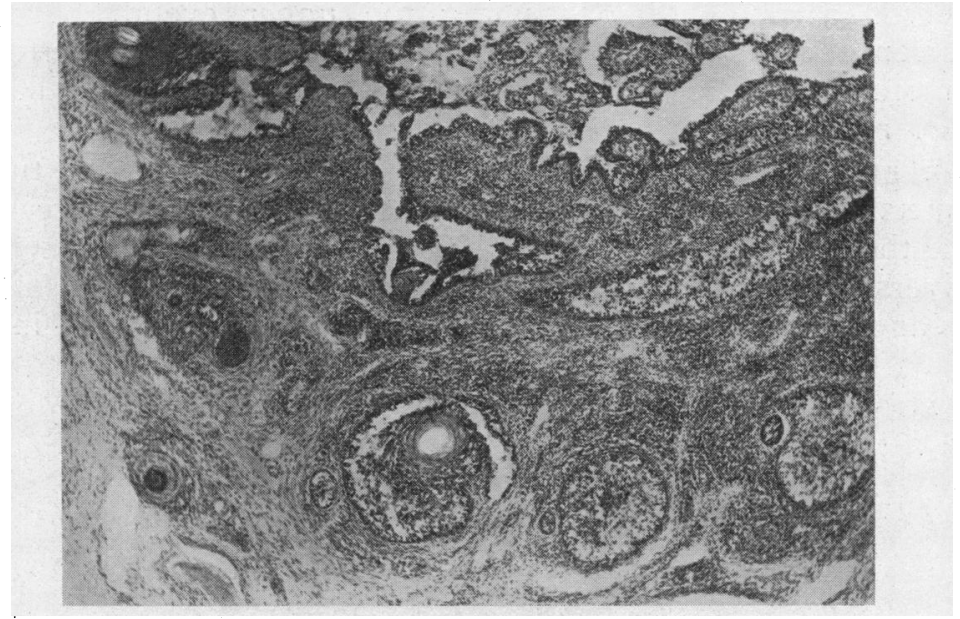

FIG. 8.

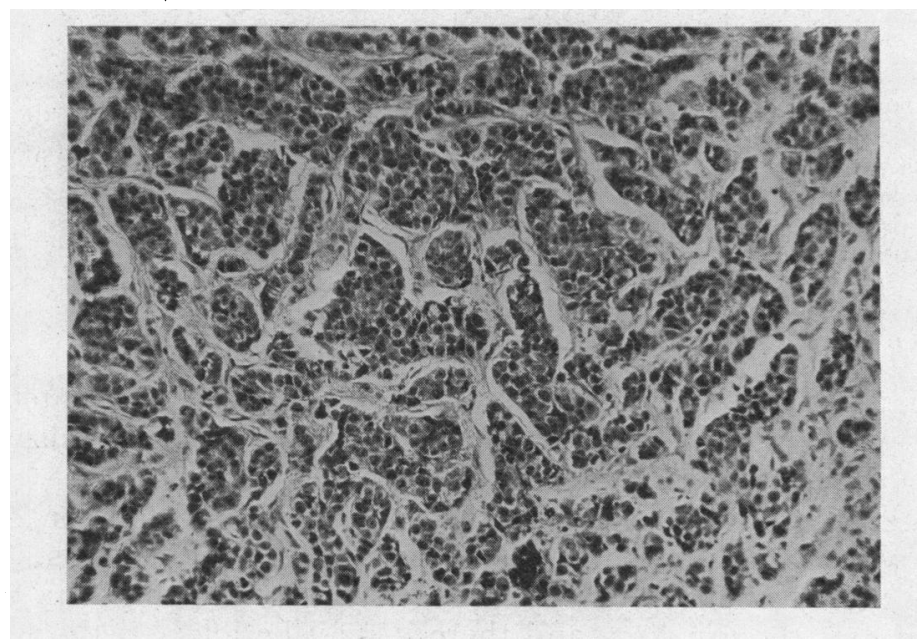

FIG O.

Abnormal cornification is totally absent. Long shoots of vacuolated cells pass into the subcutaneous tissue often surrounding hairs. If they grow in the tissue under the epithelium they may suggest an adenoid growth. The picture (Fig. 8) resembles that of basal cell carcinoma, given in Ewing's* textbook (Fig. 434).

*Ewing, James. Neoplastic Diseases. W. 13. Saunders. London, 1931. 
The small tumour of the orbit which could be felt on clinical examination consisted of solid infiltrating strands of epithelial cells : carcinoma simplex (Fig. 9). A malignant blastomatous growth seemed probable from the anomalies previously described, it became fairly certain from the recurrence as a subcutaneous tumour. However, only the histological aspect of this latter tumour allows one to make the diagnosis carcinoma with certainty.

This carcinoma is interesting on account of its being associated with lesions often called pre-carcinomatous, not described hitherto in ophthalmology. The fact that the pathological condition of the epithelium is generally restricted to the deeper layers, the absence of dyskeratosis, akanthosis and hyaline degeneration, the rare occurrence of mitosis, the association with affection of the Meibomian and sebaceous glands are in favour of the diagnosis " Paget's disease." The intensively staining homogeneous nuclei (clumping cells), though generally regular and round, the occasional appearance of large hydropic cells with pyknotic nuclei, the rare areas where the whole epithelium is very irregular remind one of " Bowen's disease." Glycogen which is richly present in Paget's and Bowen's disease could not be demonstrated, probably on account of the material being fixed in 10 per cent. formalin in which the glycogen is generally dissolved. The Sudan test in frozen sections was negative.

It is difficult to judge whether the affection originated from the skin of the eyelid which was left in situ in the first operation, which, on clinical inspection, did not show anything abnormal, or from the modified epithelium covering the posterior surface of the eyelid. As far as I know chronic trachoma and the treatment of trachoma have never been described as related to the development of carcinoma. Only Ischreyt has described a case of carcinoma in a patient affected by trachoma. In certain chronic inflammatory diseases and after prolonged traumatism, blastomatous growth may occur. Of course a third possibility, the growing from a gland or duct with secondary subepithelial growth is possible. There has been much discussion on this subject in Paget's disease of the nipple. This case was too far advanced to give any reliable indication as to the point of origin.

\section{Summary}

A case of Paget's disease (in some features resembling Bowen's disease) of the eyelid is described, which was found histologically in the eyelids removed from a patient affected with trachoma and xerosis. One year later the appearance of a subcutaneous nodule necessitated exenteration of the orbit. On histological examination this nodule proved to be a carcinoma simplex. 